\title{
FORUM
}

\section{Energiepolitik während der deutschen EU-Ratspräsidentschaft}

\author{
Michael Glos
}

\section{Rahmenbedingungen}

Die globalen Rahmenbedingungen für die Energiepolitik haben sich in den letzten Jahren erheblich gewandelt. Nach Szenarien der Internationalen Energieagentur (IEA) steigt die weltweite Nachfrage nach Energie bei Fortschreibung der gegenwärtigen Energiepolitik bis 2030 um mehr als 50 Prozent. Gleichzeitig gibt es aber einen globalen Investitionsrückstand bei Förder-, Transport- und Raffineriekapazitäten. Hinzu kommt die zunehmende Konzentration der Öl- und Gasvorräte auf wenige Versorgungsräume. Circa 70 Prozent der konventionellen Weltölreserven und circa 68 Prozent der Welterdgasreserven liegen dabei innerhalb einer ,Strategischen Ellipse', die sich vom Nahen Osten bis nach Nordwestsibirien erstreckt. Es verwundert dabei nicht, dass Energie in manchen Ländern auch zunehmend als politisches Druckmittel eingesetzt wird.

Wachsende Energienachfrage und Versorgungsrisiken bedeuten auch, dass die Preisrisiken vor allem bei Öl und Gas wachsen. Bei Kohle und Uran gibt es solche Probleme nicht.

Als energierohstoffarmes Land ist Deutschland in hohem Maße auf Energieimporte angewiesen. Eine Strategie, die Importrisiken verringert, sollte daher ein zentrales Anliegen sein. Dies liegt auch im Interesse der Wettbewerbsfähigkeit unserer Volkswirtschaft.

\section{Klimaschutz}

Neben diesen Veränderungen auf den Märkten und den steigenden Abhängigkeiten in der Versorgung wird der Klimaschutz immer wichtiger. Dabei gilt: Eine Klimaschutzpolitik, die nur von wenigen Ländern getragen wird, ist ökologisch wirkungslos und für die handelnden Staaten ökonomisch verhängnisvoll. Klimaschutzpolitik verlangt globales Handeln. Das bedeutet, dass sich auch große Länder wie die USA, China und Indien entsprechend engagieren müssen. Der Klimaschutz ist zu wichtig, als dass wir es uns leisten könnten, weiter weltweit ineffizient voranzugehen. Wir müssen uns unserer klimapolitischen Verantwortung stellen. Ganz oben auf der Agenda stehen dabei die Maßnahmen, die erfolgreich sind, ohne dabei die wirtschaftliche Leistungsfähigkeit zu gefährden. Eine Konsequenz daraus ist, dass wir bereits beschrittene Pfade selbstkritisch hinterfragen müssen. Auf Deutschland bezogen heißt das, dass der Beitrag der Kernenergie zu Versorgungssicherheit, Wirtschaftlichkeit und Klimaschutz auf absehbare Zeit nicht verzichtbar ist.

Die Kernenergie erspart uns in Deutschland bis zu 150 Millionen Tonnen an $\mathrm{CO}_{2}$-Ausstoß. Außerdem ist sie deutlich preiswerter als Gaskraftwerke oder Erneuerbare Energien. Und nicht nur dies: Mit verstärktem Einsatz von Gas zur Elektrizitätserzeugung würden wir auch unsere Importabhängigkeiten erhöhen. Wenn wir Klimaschutz national und weltweit ernst nehmen, müssen wir auch beim Thema Kernenergie konsequent sein.

* Michael Glos, Bundesminister für Wirtschaft und Technologie, Berlin. 


\section{Europäischer Energiebinnenmarkt}

International wettbewerbsfähige Energiepreise sind eine essentielle Voraussetzung für mehr Wachstum und Beschäftigung in Europa. Deshalb ist es ein wichtiges Anliegen, geeignete Rahmenbedingungen für wettbewerbsfähige Energiepreise für Industrie und Verbraucher zu schaffen. Dies wird auch während der deutschen EU-Ratspräsidentschaft ein wichtiges Ziel sein.

Wir setzen vor allem auf vier Aktionsfelder. Wir wollen den europäischen Binnenmarkt für Strom und Gas vollenden und mögliche Missbräuche von Marktmacht unter Kontrolle bringen. Wir wollen eine nach Energieträgern, Lieferquellen und Transportwegen diversifizierte Versorgung mit Energierohstoffen. Wir wollen Abhängigkeiten und Preisrisiken durch eine Erhöhung der Energieeffizienz entgegen wirken. Wir wollen einen wirtschaftlich effizienten Ausbau der regenerativen Energien, um auch auf die langfristigen Herausforderungen der Energieversorgung gut vorbereitet zu sein. Die deutsche EU-Ratspräsidentschaft bietet uns die besondere Möglichkeit, diese Vorstellungen auf europäischer Ebene voran zu treiben.

Die Europäische Kommission wird am 10. Januar 2007 ein Energiepaket und eine erstmalige Begutachtung der Energiestrategie vorlegen, die Vorschläge enthalten wird, wie die „Energiepolitik für Europa“ zukünftig gestaltet werden kann. Es wird eine der Kernaufgaben der deutschen Ratspräsidentschaft sein, daraus für den Europäischen Rat im März 2007 einen nach Prioritäten gestaffelten Energie-Aktionsplan zu entwickeln. Diesen Aktionsplan wird der Energieministerrat am 15. Februar 2007 vorbereiten.

Wir wollen den Binnenmarkt für Strom und Gas besser zum Laufen bringen. Die Binnenmarktrichtlinien für Strom und Gas sehen vor, dass spätestens zum 1. Juli 2007 der Stromund Gasmarkt in der EU allen Verbrauchern offen stehen muss. Es muss dafür gesorgt werden, dass in allen Mitgliedstaaten tatsächlich einheitliche Bedingungen für die Energiewirtschaft und die Energieverbraucher geschaffen werden. Die nationalen Teilmärkte müssen im Interesse von Verbrauchern und Industrie zu einem funktionierenden europäischen Gesamtmarkt zusammenwachsen. Dafür müssen die Voraussetzungen für eine Intensivierung des grenzüberschreitenden Handels weiter verbessert werden. Gleichzeitig ist dafür zu sorgen, dass neue Kraftwerksbetreiber in allen EU-Ländern Anspruch auf einen diskriminierungsfreien Anschluss an die Netze erhalten. Der Umsetzungsbericht der Europäischen Kommission zum zweiten Binnenmarktpaket und die Ergebnisse der Sektorenüberprüfung werden zeigen, wo die Mitgliedstaaten noch Nachholbedarf haben. Sollten Barrieren für einen europäischen Energiebinnenmarkt und für den Wettbewerb identifiziert werden, so werden wir Maßnahmen anstoßen, um diese zu beseitigen. Durch konsequente Umsetzung des bestehenden Regelungsrahmens wollen wir schnelle und sichtbare Erfolge für den Binnenmarkt und die Stromverbraucher erzielen.

Grundsätzlich sehe ich drei Handlungsfelder: Erstens sollte die Zusammenarbeit und Unabhängigkeit nationaler Regulierungsbehörden verbessert werden. Hier geht es vor allem um grenzüberschreitende Sachverhalte. Der Netzbereich muss als neutraler Marktplatz für alle Nutzer zur Verfügung stehen. Wir benötigen Investitionen in die Erhaltung und den Ausbau der Netze, wenn ein funktionsfähiger europäischer Binnenmarkt in naher Zukunft Realität werden soll. Wir brauchen die Netzinvestitionen vor allem auch, damit neue Anbieter ihre Kraftwerksprojekte realisieren können. Dies wird den Wettbewerb beleben. Drittens brauchen wir mehr Markttransparenz. In allen europäischen Staaten ist die Stromerzeugung in der Hand weniger Unternehmen oder gar in der Hand eines Unternehmens konzentriert. Diese Unternehmen verfügen über marktrelevante Informationsvorsprünge. Um die Voraus- 
setzungen für Wettbewerb zu verbessern, sollte im Bereich der Stromerzeugung für mehr Transparenz durch die Veröffentlichung von relevanten Kraftwerksdaten gesorgt werden.

Bei alledem ist es wichtig, dass die bestehenden Regeln in allen Mitgliedstaaten wirkungsvoll umgesetzt werden. Wenn auch dann noch Defizite bestehen, muss über weitere Maßnahmen nachgedacht werden.

\section{Energieaußenbeziehungen}

Ein weiterer Schwerpunkt der deutschen EU-Ratspräsidentschaft sind die Energieaußenbeziehungen. Wir wollen die Rahmenbedingungen für Investitionen weltweit - auch in politisch schwierigen Ländern - verbessern. Wenn uns das gelingt, dann können wir auch die Preisrisiken mildern und dienen damit dem Wirtschaftsstandort Europa. Dabei geht es auch um stärkere Verflechtungen vor allem mit den Produzenten-Ländern. Denn gegenseitige Abhängigkeiten bringen auch mehr Sicherheit. Wir werden die deutsche Präsidentschaft gezielt zum Dialog und zur Kooperation der EU mit den wichtigen Förder-, Transit- und Verbraucherländern nutzen. Die EU muss dort mit einer Stimme sprechen, wo die Mitgliedstaaten gemeinsame Interessen haben. Wichtige Elemente sind aus meiner Sicht die Verankerung der Energiezusammenarbeit der EU und Russlands im Rahmen eines Nachfolgeabkommens zum eines neuen Partnerschafts- und Kooperationsabkommens und die Ausdehnung der Energiegemeinschaft Südosteuropa. Damit exportieren wir unser Regelwerk und sorgen für sichere Rahmenbedingungen. Wenn wir erfolgreiche Außenbeziehungen wollen, brauchen wir auch eine Diskussion zur Klärung der Arbeitsteilung hinsichtlich der Aufgaben der Unternehmen, der Mitgliedstaaten und der EU. Die wirtschaftliche und politische Stärke Europas darf nicht durch ein Durcheinander bei der Vertretung gemeinsamer Interessen geschmälert werden.

\section{Energieeffizienz}

Energieeffizienz ist ein Thema, bei dem es viel Nachholbedarf gibt. Auf europäischer Ebene geht es beispielsweise um eine konsequente Umsetzung der Ökodesign-Richtlinie einsetzen. Der am 20. Oktober 2006 veröffentlichte Aktionsplan Energieeffizienz der EUKommission bietet für die Diskussion eine gute Basis. Wichtige Elemente dabei sind unter anderem wirksame Maßnahmen zur Verringerung der Standby-Verluste von Geräten, eine schnellere Anpassung der Energieverbrauchskennzeichnung und von Höchstverbrauchsregelungen an den wirtschaftlichen Stand der Technik, die Erschließung zusätzlicher Potenziale im Gebäudebereich sowie auch Verbesserungen bei Automobilen, ohne dabei die Leistungsfähigkeit der Automobilindustrie zu schmälern. Darüber hinaus ist auch ein internationales Energieeffizienzabkommen in der Diskussion.

\section{Erneuerbare Energien}

Marktfähige erneuerbare Energien leisten einen wichtigen Beitrag zur Weiterentwicklung des Energiemix. Ihr kosteneffizienter Ausbau ist ein wichtiges Ziel für die Zukunft. Bestehende Ziele sollten vollständig umgesetzt werden. Der von der Europäischen Kommission im Januar als Teil des Energiepaketes erwartete Fahrplan Erneuerbare Energien wird auch die Basis dafür bieten, sich auf eine Zielmarke über 2010 hinaus zu verständigen.

\section{Energieforschung}

Über diese vier Schwerpunkte unserer Präsidentschaft hinaus ist für mich die Energieforschung ein zentrales strategisches Element der Energiepolitik. Europa kommt vor allem 
durch Innovation, durch Forschung und Entwicklung und durch die Anwendung neuer Technologien ökonomisch und ökologisch voran. Das stärkt die Ziele Wettbewerbsfähigkeit, Versorgungssicherheit und Umweltverträglichkeit bereits vom Ansatz her. Forschungsrahmenprogramm aufgenommen hat. Die Optionen für saubere fossile Stromerzeugung hinsichtlich Abscheidung und Lagerung von $\mathrm{CO}_{2}$ sollten zu einem Schwerpunkt der Forschung werden. Denn wir müssen erst über die Möglichkeiten, aber auch über die Kosten informiert sein, bevor wir den Unternehmen Vorgaben machen. Dabei muss es um einen breiten Ansatz gehen, der den Energieträgermix nicht verzerrt. Es geht also um saubere fossile Energieträger und nicht nur um saubere Kohle.

\section{Mehr Rationalität!}

Die Herausforderungen in der Energiepolitik sind groß. Deshalb kann es nicht verwundern, dass die energiepolitische Diskussion in Europa sehr kontrovers geführt und von starken Emotionen geleitet wird. Ein Anliegen der Energiepolitik muss es dennoch sein, mehr Rationalität in die Debatte zu bringen. Und das wird auch die Leitlinie während der deutschen Ratspräsidentschaft sein.

Vor diesem Hintergrund werden wir auch die deutsche G8-Präsidentschaft dazu nutzen, das Thema Klimawandel und im Energiebereich das Thema Energieeffizienz auf internationaler Ebene, auch im Dialog mit den wichtigsten Schwellenländern, voran zu bringen.

Wir haben 2007 ein umfangreiches Arbeitsprogramm vor uns, insbesondere mit Blick auf den Energie-Aktionsplan des Frühjahrsgipfels 2007. Gemeinsam können wir der Energiepolitik in Europa wichtige Impulse geben!

Der Beitrag entstand im Rahmen des am Institut für Europäische Politik gemeinsam mit der ASKO EUROPA-STIFTUNG durchgeführten Projekts „Ein Europa der Bürger - Verfassung und effiziente Politik. “

\section{The History of European Integration}

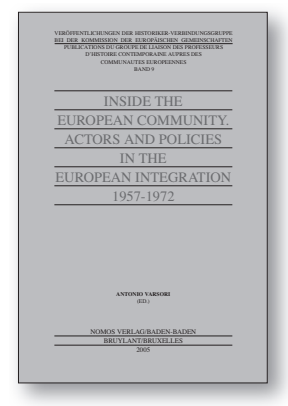

\section{Inside the European Community.}

Actors and Policies in the European Integration 1957-1972

Von Antonio Varsori

2006, 483 S., brosch., 98,- €, ISBN 978-3-8329-1191-1

(Veröffentlichungen der Historiker-Verbindungsgruppe

bei der Kommission der EG, Bd. 9)

The Rome Treaties established new actors in European policies which played autonomous roles in implementing the customs union and the common agricultural policy and paved the way for further dimensions of European integration. 